\title{
MediQuit, an Electronic Deprescribing Tool for Patients on Polypharmacy: Results of a Feasibility Study in German General Practice
}

\author{
Ulrike Junius-Walker ${ }^{1}\left(\right.$ ) Annika Viniol ${ }^{2} \cdot$ Matthias Michiels-Corsten $^{2} \oplus \cdot$ Navina Gerlach $^{2} \oplus$. \\ Norbert Donner-Banzhoff ${ }^{2}{ }^{-1} \cdot$ Tanja Schleef $^{1}$
}

Accepted: 15 April 2021 / Published online: 12 July 2021

(c) The Author(s) 2021

\begin{abstract}
Background Deprescribing is an important task for general practitioners (GPs) in the face of risky polypharmacy. The electronic tool "MediQuit" was developed to guide GPs and patients through a deprescribing consultation that entails a drug-selection phase, shared decision making, and advice on safe implementation.

Objectives A pilot study was conducted to determine the target group of patients that is selected for consultation and to assess the impact, patient involvement, and feasibility of the tool.

Methods This was an uncontrolled pilot study. GPs from two German regions were invited to use MediQuit in consultations with a view to deprescribing one drug, if appropriate. They selected patients on the basis of broad inclusion criteria. Collected data entailed participants' characteristics, patients' medication lists, deprescribed drugs, and feasibility assessments. Patients were contacted shortly after the consultation and again after 4 weeks.

Results In total, 16 GPs agreed to participate, of whom ten actually performed deprescribing consultations. They selected 41 predominately older patients on excessive polypharmacy. Deprescribing was achieved in $70 \%$ of consultations in agreement with patients. Drugs deprescribed were symptom-lowering and preventive drugs (mainly anatomical therapeutic chemical classes A and C). GPs found MediQuit useful in initiating communication on this issue and enhancing deliberations for a deprescribing decision. The median consultation length was $15 \mathrm{~min}$ (interquartile range 10-20). At follow-up, GPs and patients infrequently disagreed on which drug(s) was discontinued, and GPs rated patient involvement higher than did patients themselves.

Discussion MediQuit assists in identifying concrete deprescribing opportunities, patient involvement, and shared decision making. The three-step deprescribing procedure is well-accepted once initial organizational efforts are overcome. After revision, further studies are needed to enhance the quality of evidence on acceptance and effectiveness.
\end{abstract}

\section{Introduction}

Deprescribing is becoming increasingly important as more and more patients with multimorbidity are on polypharmacy. Polypharmacy may be inappropriate and potentially leads to adverse drug events, frailty, increased emergency care, hospital admissions, and mortality [1,2]. Deprescribing counteracts overprescribing and is defined as the process of

Ulrike Junius-Walker

junius-walker.ulrike@mh-hannover.de

1 Institute of General Practice, Hannover Medical School, Hannover, Germany

2 Institute of General Practice, Marburg University, Marburg, Germany

\section{Key Points}

The digital tool MediQuit facilitates patient-centered deprescribing for patients on polypharmacy.

General practitioners thought the tool promoted patients' understanding of the advantages and disadvantages of deprescribing a drug candidate and helped with shared decision making.

A deprescribing consultation with MediQuit does not replace a comprehensive medication review, but it does provide an opportunity for general practitioners to approach this difficult issue. 
withdrawal of an inappropriate medication, supervised by a health care professional with the goal of managing polypharmacy and improving outcomes [3]. Yet physicians find it difficult to deprescribe as decisions need to be based on complex interacting factors, and medical evidence is often uncertain [4]. Hence, research expands to examine ways on how to guide doctors in deprescribing decisions.

There are two principally different deprescribing methods [5]. One offers recommendations on specific medications to be avoided (or considered) for older patients, such as the Beers criteria, the FORTA (Fit For The Aged) list, and the STOPP (Screening Tool of Older Persons' Prescriptions)/ START (Screening Tool to Alert to Right Treatment) criteria, so-called explicit criteria [6-8]. The second method assesses the appropriateness of patients' medication using implicit criteria that guide the clinician through a decision pathway. At each decision node, doctors make judgments by applying their own knowledge. Since these tools are usable irrespective of disease or drug classes, they are referred to as "generic." Increasingly, doctors are resorting to electronic decision aids. They provide fast information access on specific drug combinations, enable effective and organized deprescribing, and allow application at point of care [9]. PrimaEDS, MedSafer, and TRIM, for example, are electronic tools that make use of patient-specific medication lists and patient-related medical data to perform a pharmacotherapeutic analysis in which indications, dosages, potential interactions, and risks are checked [10-12]. Other electronic tools make provisions for steps that assist with gathering a patient's perspective on the purpose of medication use [13, 14].

Beyond the achievements of existing aids, we intended to develop a tool that supports a "deprescribing consultation," defined as a consultation in which deprescribing opportunities are identified, discussed, and decided upon, involving patients as much as possible. It has been recognized that shared decision making and a thorough monitoring process are underused in deprescribing, and solutions on how to support doctors with this complex issue are called for [15]. Following the Medical Research Council guidance for developing complex interventions [16], we undertook a systematic review on deprescribing in primary care [5] and held focus groups with general practitioners (GPs), specialist doctors, and pharmacists to discuss deprescribing and expectations in their work context [17]. Patients were also interviewed to account for their perspectives and needs. One important finding was to view stopping medicines as an open-ended experiment that may be abandoned, if necessary, an observation also made by Reeve et al. [18].

\subsection{Development of the MediQuit Program}

Based on these premises, we developed an electronic tool, named MediQuit. It is designed to guide doctors through three steps of deprescribing during consultation. In step I, GPs examine patients' current medication charts and follow an algorithm of questions to identify a medication potentially suitable for deprescribing. Step II supports doctor-patient communication in two ways. First, the software suggests verbal prompts and phrases that GPs can use when addressing the topic of deprescribing. Second, the software offers a range of arguments on the benefits and risks of drug discontinuation, which doctors and patients can pick and discuss to prepare for a shared decision. Once a mutual decision is made, step III equips doctors and patients for the discontinuation process. At this stage, the program provides tapering information and a semiautomatically generated take-home information sheet for the patients on how to monitor their health and manage uncertainties in the withdrawal phase. Prior to the pilot test, MediQuit underwent a panel test. Advice was sought from experts in the fields of geriatrics, pharmacology, general practice, and patient orientation to improve its content and comprehensibility. ${ }^{1}$

\subsection{Objectives of the Pilot Test}

A pilot study was conducted to test this new tool with the aim of gaining information on feasibility, acceptance, and impact. We intended to receive answers to the following specific questions:

1. What are the characteristics of patients who are selected by GPs to receive a deprescribing consultation?

2. To what extent does the use of MediQuit result in reducing or stopping medication?

3. How do GPs and patients evaluate MediQuit in terms of usefulness, facilitation of shared decision making, and adequacy of consultation length?

\section{Methods}

\subsection{Training and Recruitment}

We contacted GPs in two German regions (Marburg and Hannover) using established relationships, either through teaching assignments or through primary care research networks. Study staff helped participating GPs to install the

\footnotetext{
${ }_{1}$ An introductory video about MediQuit is available in German at https://arriba-hausarzt.de/module/mediquit.
} 
program on their practice computers. A 30-min training session included a demonstration of the program with its deprescribing steps I-III and instructions on the use of the built-in links for specific withdrawal recommendations. GPs received a user manual and a case vignette to familiarize themselves with all aspects of the program and the deprescribing process. Additional telephone support was given where necessary.

GPs were invited to recruit three to five patients each, using the following inclusion criteria: patients had to be at least 60 years of age, take five or more different medicines per day (polypharmacy), and have at least three chronic diseases, with one of cardiovascular origin. Exclusion criteria were cognitive impairment, insufficient language skills, no access to practice computer (e.g., home visits and care facilities), and lack of patient consent. GPs recruited potential patients prospectively by screening their surgery schedule each morning before the first consultation.

\subsection{Ethical Considerations}

GPs and patients gave written and informed consent for participation in the study. In the recruitment process, patients were advised that they would receive a consultation that focused on their medication regimen with a view to discussing potentially dispensable medication. Patient data used in the deprescribing software were not stored. The Ethics Committees of Hannover Medical School (No. 2326-2014) and the Department of Medicine, Marburg University (No. 160/15), gave approval for the study.

\subsection{Intervention: MediQuit Deprescribing Consultation}

GPs were asked to conduct the deprescribing consultation in conformity with the three MediQuit steps. They were asked to print out the current and complete medication list in advance of the consultation and, after the consultation, to hand out the individualized monitoring information sheet and the updated medication list. A follow-up appointment was arranged if deemed necessary.

\subsection{Data Collection and Analysis}

GPs completed patient-related questionnaires after each deprescribing consultation. The study team interviewed participating patients by telephone $2-4$ days (T1) and 4 weeks (T2) after the deprescribing consultation. Questionnaires and interviews included the following items:

- GP questionnaire
1. Patient health data (frailty state using SOF [Study of Osteoporotic Fractures] [19], list of chronic diseases) and medication list

2. Deprescribing consultation: the deprescribed medication and mode of deprescription, involvement in the decision

3. Acceptance and feasibility of the MediQuit tool and the deprescribing consultation with its three phases

- Patient questionnaire (at T1)

1. Patient characteristics: age, sex, family status, years in GP's care, care dependency status, independence in activities of daily living, independence with taking medicines

2. Deprescribing consultation: the deprescribed medication and mode of deprescription (e.g., tapering over 2 weeks), satisfaction with receiving and sharing information, involvement in the decision

3. Acceptance and feasibility of the consultation (including duration) and the computer-aided MediQuit program

4. Details on the planned monitoring for deprescribed drugs

- Patient questionnaire (at T2)

1. Sustained deprescription: medication name and mode of deprescription

2. Experiences with the implementation and monitoring of the deprescribed drugs

3. Health and medication changes during the 4 weeks after the deprescribing consultation

4. Sustained satisfaction with the decision

Pseudonymized data were entered into a secure webbased data form (GCP certified system SecuTrial $\left.{ }^{\circledR}\right)^{2}$. Data were exported into IBM SPSS Statistics 26 and analyzed descriptively. We used inferential statistics to analyze patient characteristics according to the outcome of the deprescribing consultation (single-factor variance analysis, Fisher exact test, $\chi^{2}$ test). For interrelated patient and doctor ratings, comparisons were made using Kappa statistics.

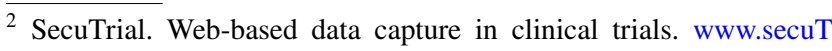
rial.com. 
Table 1 Patient characteristics

\begin{tabular}{|c|c|c|c|c|c|}
\hline Characteristics $(N=41)$ & All & $\begin{array}{l}\text { With deprescription } \\
(\mathrm{N}=26)\end{array}$ & $\begin{array}{l}\text { Remaining on drugs } \\
(N=4)\end{array}$ & $\begin{array}{l}\text { With disagreement on } \\
\text { decision }(N=7)^{\mathrm{a}}\end{array}$ & $p$ value \\
\hline Age, years & $77.1 \pm 8.1$ & $75.8 \pm 7.7$ & $71.5 \pm 9.5$ & $79.4 \pm 4.8$ & 0.25 \\
\hline Female sex & 61.0 & 62.5 & 50.0 & 57.1 & 0.90 \\
\hline Frailty according to SOF & 30.0 & 28.0 & 0.0 & 28.6 & 0.72 \\
\hline Care dependency status $^{\mathrm{b}}$ & 20.0 & 15.4 & 0.0 & 28.6 & 0.62 \\
\hline Number of diagnoses & $8.2 \pm 2.2$ & $8.1 \pm 1.9$ & $7.1 \pm 2.0$ & $8.3 \pm 2.5$ & 0.51 \\
\hline Number of medications ${ }^{c}$ & $10.0 \pm 3.6$ & $10.0 \pm 3.1$ & $9.3 \pm 0.5$ & $8.6 \pm 2.5$ & 0.52 \\
\hline
\end{tabular}

Data are presented as $\%$ or mean \pm standard deviation unless otherwise indicated

GP general practitioner, SOF Study of Osteoporotic Fractures

${ }^{a}$ Disagreement between GP and patient on what happened in the deprescribing consultation

${ }^{\mathrm{b}}$ Care dependency status classified by the health insurance fund

${ }^{\mathrm{c}}$ Including on-demand medication

\section{Results}

\subsection{Recruitment of General Practitioners and Selection of Patients}

In total, 16 GPs agreed to participate and received the MediQuit training. Six GPs did not manage to recruit any patients. They mainly found it too time consuming to broach the subject with patients and organize deprescribing consultations. In one case, software upload was incomplete; in another case, the GP had too many patients with insufficient language skills. The ten actively recruiting GPs were on average $48 \pm 9$ years old and had a mean of $20 \pm 9$ years of experience in practice. Four GPs were male. Four GPs worked in a single-handed practice. Practices were located in rural and urban settings.

GPs chose patients on the basis of the broad inclusion criteria and approached them during their scheduled consultation. GPs tended to target older patients and those with a high degree of multimorbidity receiving a substantial number of different prescriptions. Details of the 41 participating patients are given in Table 1, including a subgroup comparison according to the deprescribing decisions (yes, no, ambiguities concerning the decision).

\subsection{Impact of the MediQuit Consultation on Deprescribing}

Of the 41 patients, 37 participated in telephone interviews (T1, T2). Four patients were lost to follow-up: one patient could not be contacted by telephone, two patients declined after having given consent, and one patient had a stroke 1 day after the deprescribing consultation, in which a lipidlowering drug was stopped.
Of the 37 patients, 26 reported stopping or tapering medications at $\mathrm{T} 1$ because of the decision made during the deprescribing consultation. Another four patients remained on their medication. The seven remaining patients could either not remember details of the deprescribing decision or reported a decision that conflicted with the GPs' records. This meant that $70 \%$ of the patients who had received a deprescribing consultation actually reduced drugs in accordance with the GPs' deprescribing consultation at T1. During the follow-up period of 4 weeks (T2), five patients resumed their medication (Fig. 1). Therefore, $57 \%$ of patients managed a sustained reduction of their medications in line with the GPs' recommendations (Fig. 1).

It is noteworthy that, in eight cases, more than one drug was discontinued or reduced in dosage as a result of the deprescribing consultation. However, the MediQuit program had specified that GPs discuss only one medication at a time in order to better control the potential effects of withdrawal.

GPs deprescribed only a certain range of drugs. Considering second-level ATC groups [20], proton pump inhibitors (PPIs) and diuretics were most frequently deprescribed followed by angiotensin II antagonists, antigout preparations, and lipid-modifying drugs. GPs and their patients therefore agreed to deprescribe both symptom-lowering and preventive drugs (Table 2).

At the 4-week follow-up (T2), four of the 26 patients who had stopped taking or reduced a drug had resumed or replaced their drugs because of re-occurring symptoms (two PPIs, two diuretics). One further patient had not started with the discontinuation process (antihypertensive), because he "wanted to finish the package." Patients who continued with the deprescribing decision felt well. However, one patient noted re-occurring symptoms after cessation of his diuretic drug. Notably, seven patients experienced additional medication changes during follow-up 


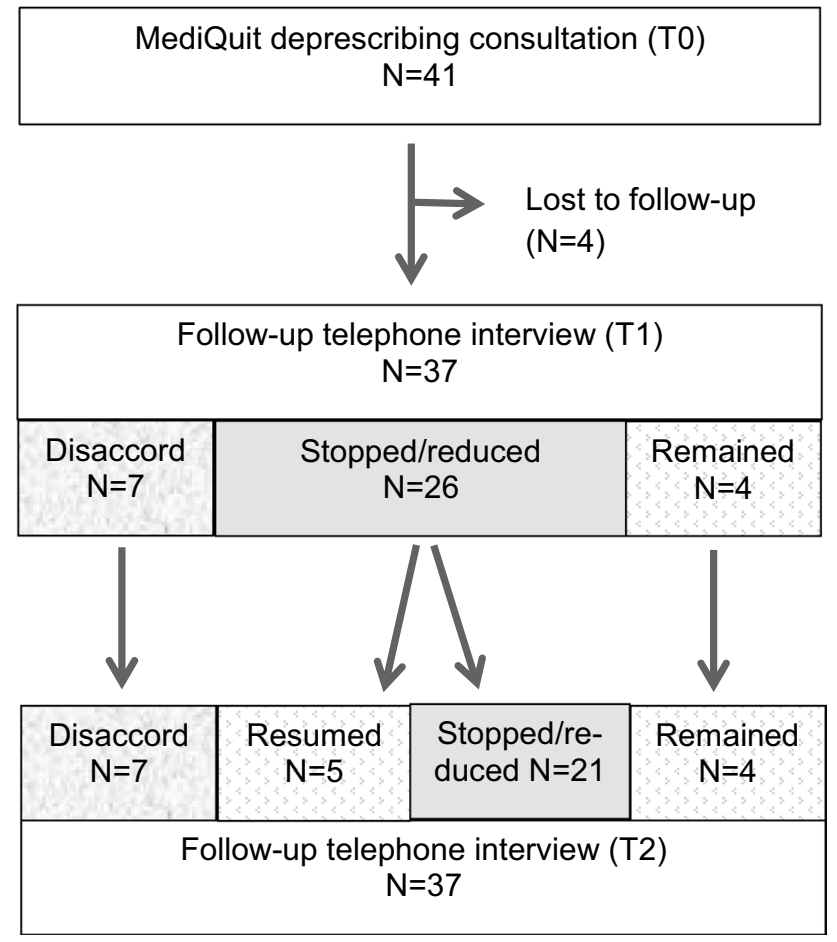

Fig. 1 Flow chart of the number of patients with MediQuit consultations and their outcomes during follow-up

unrelated to the deprescribing consultations. Reasons were hospital stays, diagnostic procedures with other medical specialists, or new health issues.

\subsection{Decision Making, Feasibility, and Acceptance}

In 32 of 37 consultations, GPs chose the drug candidates potentially suitable for deprescription. In 36 consultations, GPs used the MediQuit algorithm in phase I; in two of these instances, the algorithm was found to be pivotal for the choice. In five instances, patients suggested a drug candidate.

In phase II of the deprescribing consultation, the aim was to reach a decision shared by the GP and the patient. When asked about the involvement in the deprescribing decision afterwards, almost $50 \%$ of patients indicated that the decision had been shared with the GP, whereas GPs stated that $70 \%$ of deprescribing decisions were shared. Doctors and patients agreed on the degree of involvement for only $43 \%$ of the deprescribing consultations $(k=-0.31)$ (Fig. 2).

GPs required a median of 15 min (interquartile range 10-20) for the deprescribing consultations using MediQuit. In their opinion, $85 \%$ of the consultations were just right in length, whereas $15 \%$ were too long. In total, 95\% of the patients appraised the duration of the consultation as appropriate.
Table 2 Deprescribed drugs according to ATC code for 26 patients from telephone interviews of patients 2-4 days after the deprescribing consultation

\begin{tabular}{clc}
\hline ATC & Designation & No. of drugs \\
\hline A & Alimentary tract and metabolism & $\mathbf{1 3}$ \\
A02 & Drugs for acid-related disorders (all PPIs) & 9 \\
A10 & Antidiabetics & 2 \\
A12 & Minerals & 2 \\
B & Blood and blood-forming organs & $\mathbf{2}$ \\
B01 & Antithrombotic agents & 2 \\
C & Cardiovascular system & $\mathbf{1 7}$ \\
C03 & Diuretics & 8 \\
C07 & Beta-blocker & 2 \\
C09 & Agents acting on RAS & 4 \\
C10 & Lipid modifying drugs & 3 \\
H & Systemic hormonal preparations & $\mathbf{1}$ \\
H03 & Thyroid therapy & 1 \\
M & Musculoskeletal system & $\mathbf{4}$ \\
M04 & Antigout preparations & 4 \\
N & Nervous system & $\mathbf{1}$ \\
N03 & Antiepileptic drugs & 1 \\
Total & & $\mathbf{3 8}$ \\
\hline
\end{tabular}

ATC anatomical therapeutic chemical, PPIs proton pump inhibitors, $R A S$ renin-angiotensin system (in this case, entirely angiotensin-II receptor blockers)

After each consultation, GPs provided feedback on the MediQuit program. GPs thought MediQuit helped them make decisions in one-half of the consultations. In two-thirds of consultations, it supported the communication and exchange of information with patients. Most frequently, MediQuit was helpful in facilitating patients' understanding of the advantages and disadvantages of medication decisions (Fig. 3).

Patients were generally well-satisfied with the deprescribing consultation. All except one (3\%) patient felt that the information received was fairly (42\%) or very $(55 \%) \mathrm{com}$ prehensible. All patients were fairly $(35 \%)$ or very $(65 \%)$ satisfied with the decisions made and with the consultation process as a whole. In total, $74 \%$ of patients liked the computer program MediQuit, whereas $26 \%$ expressed no firm opinion. After 4 weeks, patients' satisfaction with the consultation remained unchanged.

\section{Discussion}

\subsection{Summary of Findings}

The electronic software MediQuit was developed to facilitate a patient-centered deprescribing consultation. It is designed to guide GPs through three phases, consisting of a selection guide for a drug candidate, a communication phase with 


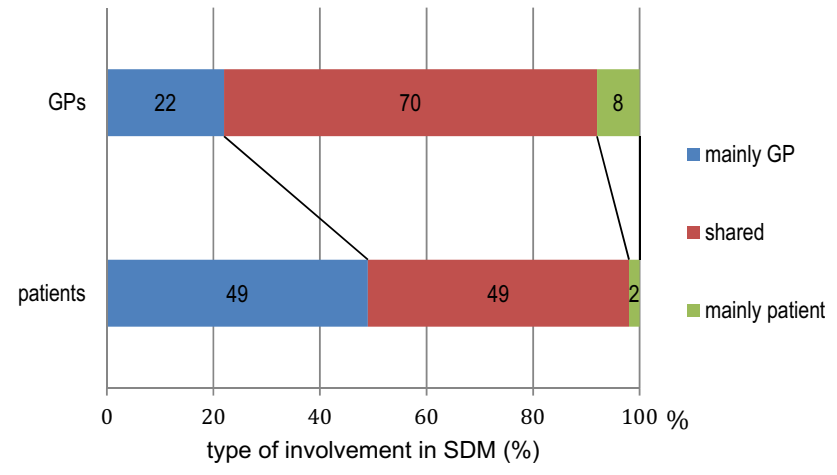

Fig. 2 Percentage of consultations with mainly GP/shared/patient involvement in decisions ( $n=37$ consultations). $G P$ general practitioner, $S D M$ shared decision making

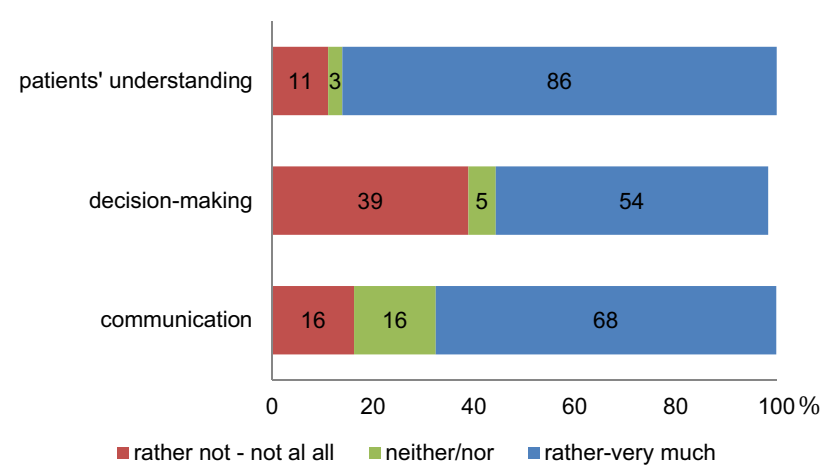

Fig. 3 General practitioner (GP) evaluations on the ability of MediQuit to facilitate three aspects of patient-centeredness (\% of consultations). GPs rated their 37 deprescribing consultations. For example, GPs thought that MediQuit facilitated patients' understanding in 32 of 37 consultations $(86 \%)$ rather or very much

shared decision making, and safe implementation and monitoring of the deprescribing decision. On the basis of broadly formulated patient inclusion criteria, GPs selected elderly patients with substantial multimorbidity and excessive polypharmacy (ten or more drugs). MediQuit satisfied expectations in regard to the deprescribing impact. In total, $70 \%$ of patients reported a cessation/withdrawal of one or more drugs in agreement with the GP; altogether, $57 \%$ of patients succeeded in a sustained withdrawal or reduction. GPs had a differentiated assessment of the usefulness of the MediQuit tool. They thought it was rarely needed to select a drug candidate for deprescription. In contrast, it was often considered helpful in supporting patient understanding, shared decision making, and implementation. Patients were well-satisfied and involved in the procedure. They generally understood the deliberations leading to a deprescribing decision.

\subsection{Findings in Light of Current Debate}

The UK National Health Service Deprescribing Guide recommends targeting patient groups who will benefit most from a deprescribing review, such as patients with multimorbidity, with palliative care needs, with excessive polypharmacy, or with frailty or a decline in hepatic or renal function [21]. Indeed, the GPs in our study selected older patients with multimorbidity and excessive polypharmacy for a deprescription consultation. When selecting drugs for deprescribing, the GPs did not stop at preventive drugs despite reluctance towards changing preventive treatment because of uncertain evidence and fear that patients may feel they are being "given-up" on [22, 23]. In a recent electronic survey from the USA, geriatricians, general physicians, and cardiologists were invited to consider deprescribing preventive drugs in different hypothetical cases. Proactive deprescribing in an older patient with no specific concerns was considered in a minority of cases and even less frequently in patients with a history of a myocardial infarction. The interprofessional variation in deprescribing was high, showing a need for more evidence and a recognition of the barriers [24].

It is not self-evident that trials aiming to optimize or reduce drugs in patients with polypharmacy actually result in a reduction of medications. Two recent randomized controlled trials in general practice reported a nonsignificant trend towards even more prescriptions [25, 26]. Our findings indicate that a deprescribing consultation may trigger a sustained reduction of drugs in patients with polypharmacy. Of course, this requires further investigation in a pragmatic controlled trial.

Many tools that aid doctors with deprescribing predominately focus on the "technical safety aspects" of a prescription review, such as avoiding drug-drug/drug-disease interactions, potentially inappropriate drugs, or an anticholinergic burden. At the same time, patient engagement plays an inferior role. GPs in this study highlighted that MediQuit was particularly helpful in communicating with their patients. It facilitated patients' understanding of the advantages and disadvantages of discontinuing medication and led to shared decision making. The World Health Organization calls on the practice of patient engagement, particularly in the field of medication management, in order to improve drug safety and people-centered health care [27]. Indeed, a variety of patient aspects may have to be considered. Patients may want to discontinue medication because they have a considerable treatment burden or worry about side effects or 
potential harms caused by polypharmacy [28]. Conversely, patients may want to continue medication because they have been told to take it ad infinitum or because they experienced beneficial effects in the past [18]. Notwithstanding patients' medication preferences, their health may have changed, and different aspects and life goals may have gained priority. Most patients are open to a reduction of their medicines. They wish to be involved in deprescribing decisions on the basis of thorough information and discussion [15].

\subsection{Strengths and Limitations}

While the strength of the MediQuit consultation seems to lie in accomplishing its objectives, in encouraging patient engagement, and in its general acceptance by doctors and patients, barriers should also be taken into account. One barrier refers to technical aspects of the computer program. Installing the software on practice computers and the time needed to familiarize oneself with the digital application of MediQuit may have deterred GPs from patient recruitment in some practices. Once in use, the lack of integration into the existing practice software prevented doctors from linking the patient's digital medication chart with MediQuit.

Another note of caution refers to the way decisions on deprescribing were perceived. Although the majority of doctors and patients stated that decision making was shared, they actually only agreed in $43 \%$ of consultations on whether both or one party was predominately involved. From the patient perspective, doctors mainly overestimated patient involvement. Previous studies on shared decision making also observed this phenomenon, which should require particular attention from doctors [29]. On the same subject of doctor-patient concordance, another issue arose in the follow-up interviews. Seven of 37 patients named a different medicine selected for deprescription than their GPs. A possible explanation is that several drugs had been deprescribed in the respective consultations, and that GPs recorded only one drug in the provided placeholder of the study form, as instructed, whereas patients remembered another. To minimize misunderstandings on which drug to stop, we are considering adding a teach-back module ("asking patients to repeat back information and instructions") to the next version of MediQuit [30].

The length of the MediQuit consultation can be viewed simultaneously as a strength and limitation. Although the brevity of the MediQuit procedure facilitates its implementation in routine practice, MediQuit cannot claim to offer a comprehensive medication review covering the underuse of medications in polypharmacy. Yet it can be repeatedly applied in the rapidly changing medication regimens that older adults encounter [31].

Some limitations have also arisen from the study design and methods. Results are based on a small convenience sample of GPs interested in testing a deprescribing tool. GPs chose patients they deemed particularly suitable. Patients with cognitive deficits or language difficulties were excluded to avoid invalid survey responses. No cases with carer involvement were encountered. The practice test consisted of a single-arm trial, which compromised interpretation of results. Finally, we did not investigate potential risks that may occur with deprescribing because the small sample size and short follow-up period of 4 weeks did not allow such activities.

\subsection{Conclusions}

A deprescribing consultation using MediQuit identified concrete deprescribing opportunities, facilitated patient engagement and shared decision making during the deprescribing process, and supported self-management in the implementation phase. Some advanced technical software solutions and content-related aspects of MediQuit require further refinement: the deprescription of more than one drug at a time, systematic assurance of patients' understanding, and the need for an agreed and written medication plan after the deprescribing consultation. The electronic decision tool focuses on a central aspect of routine care that is associated with much uncertainty. Further studies with larger samples and analytical designs are required to confirm its utility.

Acknowledgments We thank T. Scheithauer from the Arriba Cooperative for creating the digital design of MediQuit. We also thank the following experts for their valuable advice: Prof. Dr. ML Dierks, Prof. Dr. W Haefeli, U Hüttner, PD Dr. med O. Krause, Dr. med PR Lubeck, Dr. med A Mainz, Dr. A Maun, Dr. med. U Popert, Prof. Dr. med P Thürmann. We thank all GPs and patients for their participation. We thank the German Research Foundation (DFG) for funding the project.

Funding Open Access funding enabled and organized by Projekt DEAL.

\section{Declarations}

Funding The project-including this pilot test-was funded by the German Research Foundation DFG, grant number: DFG DO 513/11-1; JU 2992/2-1.

Conflicts of interest Ulrike Junius-Walker, Annika Viniol, Matthias Michiels-Corsten, Navina Gerlach, Norbert Donner-Banzhoff, Tanja Schleef have no conflicts of interest that are directly relevant to the content of this article.

Ethics approval The Ethics Committees of Hannover Medical School (No. 2326-2014) and the Department of Medicine, Marburg University (No. 160/15) gave approval for the study. Data protection considerations were included and approved. The study was performed in accordance with the ethical standards as laid down in the 1964 Declaration of Helsinki and its later amendments or comparable ethical standards.

Consent to participate All participants of the study gave written informed consent. 
Consent for publication Not applicable.

Availability of data and material The datasets generated during and/or analyzed during the current study are available from the corresponding author on reasonable request.

Authors' contributions All authors made substantial contributions to the conception or design of the work; or the acquisition, analysis, or interpretation of data; or the creation of new software used in the work. UJW drafted the work. AV, MMC, NG, and TS revised it critically for important intellectual content. All authors approved the version to be published and agreed to be accountable for all aspects of the work in ensuring that questions related to the accuracy or integrity of any part of the work are appropriately investigated and resolved.

Open Access This article is licensed under a Creative Commons Attribution-NonCommercial 4.0 International License, which permits any non-commercial use, sharing, adaptation, distribution and reproduction in any medium or format, as long as you give appropriate credit to the original author(s) and the source, provide a link to the Creative Commons licence, and indicate if changes were made. The images or other third party material in this article are included in the article's Creative Commons licence, unless indicated otherwise in a credit line to the material. If material is not included in the article's Creative Commons licence and your intended use is not permitted by statutory regulation or exceeds the permitted use, you will need to obtain permission directly from the copyright holder. To view a copy of this licence, visit http://creativecommons.org/licenses/by-nc/4.0/.

\section{References}

1. Wastesson J, Morin L, Tan E, Johnell K. An update on the clinical consequences of polypharmacy in older adults: a narrative review. Expert Opin Drug Saf. 2018;17(12):1185-96.

2. Khezrian M, McNeil CJ, Murray AD, Myint PK. An overview of prevalence, determinants and health outcomes of polypharmacy. Ther Adv Drug Saf. 2020;11:1-10.

3. Reeve E. Deprescribing tools: a review of the types of tools available to aid deprescribing in clinical practice. Ger Ther Rev. 2020;50:98-107.

4. Cullinan S, Hansen CR, Byrne S, O'Mahony D, Kearney P, et al. Challenges of deprescribing in the multimorbid patient [published correction appears in Eur J Hosp Pharm. 2017 Sep;24(5):312]. Eur J Hosp Pharm. 2017;24(1):43-6.

5. Michiels-Corsten M, Gerlach N, Schleef T, Junius-W U, Donner-Banzhoff N, et al. Generic instruments for drug discontinuation in primary care: a systematic review. Br J Clin Pharmacol. 2020;86(7):1251-66.

6. Campanelli CM. American Geriatrics Society updated Beers Criteria for potentially inappropriate medication use in older adults. J Am Geriatr Soc. 2012;60(4):616-31.

7. Pazan F, Weiss C, Wehling M. FORTA. The FORTA (Fit fOR The Aged) list 2018: third version of a validated clinical tool for improved drug treatment in older people. Drugs Aging. 2019;36(5):481-4.

8. O'Mahony D. STOPP/START criteria for potentially inappropriate medications/potential prescribing omissions in older people: origin and progress. Expert Rev Clin Pharmacol. 2020;13(1):15-22.

9. Scott IA, Pillans PI, Barras M, Morris C. Using EMR-enabled computerized decision support systems to reduce prescribing of potentially inappropriate medications: a narrative review. Ther Adv Drug Saf. 2018;9(9):559-73.
10. Rieckert A, Sommerauer C, Krumeich A, Sönnichsen A. Reduction of inappropriate medication in older populations by electronic decision support (the PRIMA-eDS study): a qualitative study of practical implementation in primary care. BMC Fam Pract. 2018;19(1):110.

11. McDonald EG, Wu PE, Rashidi B, Forster AJ, Huang A, et al. The MedSafer Study: a controlled trial of an electronic decision support tool for deprescribing in acute care. J Am Geriatr Soc. 2019;67:1843-50.

12. Niehoff KM, Rajeevan N, Charpentier PA, Miller PL, Goldstein $\mathrm{MK}$, et al. Development of the tool to reduce inappropriate medications (TRIM): a clinical decision support system to improve medication prescribing for older adults. Pharmacotherapy. 2016;36:694-701.

13. Kouladjian O'Donnell L, Sawan M, Reeve E, Chen T, et al. Implementation of the Goal-directed Medication review Electronic Decision Support System (G-MEDSS)@ into home medicines review: a protocol for a cluster-randomised clinical trial in older adults. BMC Geriatr. 2020;20:51.

14. Drenth-van Maanen AC, Leendertse AJ, Jansen PAF, Knol W, Keijsers CJP, et al. The systematic tool to reduce inappropriate prescribing (STRIP): combining implicit and explicit prescribing tools to improve appropriate prescribing. J Eval Clin Pract. 2018;24:317-22.

15. Jansen J, Naganathan V, Carter SM, McLachlan AJ, Nickel B, et al. Too much medicine in older people? Deprescribing through shared decision making. BMJ. 2016;353:i2893.

16. O'Cathain A, Croot L, Duncan E, Rousseau N, Sworn K, et al. Guidance on how to develop complex interventions to improve health and healthcare. BMJ Open. 2019;9:e029954.

17. Gerlach N, Michiels-Corsten M, Schleef T, Junius-Walker U, Krause $\mathrm{O}$, et al. Professional roles of general pratitioners, community pharmacists and specialist providers in collaborative drug deprescribing - a qualitative study. BMC Fam Pract. 2020;21:183.

18. Reeve E, Low LF, Hilmer SN. Beliefs and attitudes of older adults and carers about deprescribing of medications: a qualitative focus group study. Br J Gen Pract. 2016;66:e552-60.

19. Ensrud KE, Ewing SK, Taylor BC, Fink HA, Cawthon PM, et al. Comparison of 2 frailty indexes for prediction of falls, disability, fractures, and death in older women. Arch Intern Med. 2008; 168:382-9.

20. BfarM. ATC-Code-Zuordnung in die Fachgebiete der Zulassungsabteilungen 2 bis 4. Stand Juli 2019. https://www.bfarm. de/DE/Arzneimittel/Arzneimittelzulassung/Service/amAktuell/ atc-code-zuordnung-nach-atc-code.html. Accessed 16 Sep.

21. Olasehinde-Williams O. NHS. Deprescribing Guide. https:// southendccg.nhs.uk/your-health-services/healthcare-profession als/medicines-management/medicines-management-resources/ 2308-deprescribing-guide/file. Accessed 16 Sep.

22. Luymes CH, Poortvliet RKE, van Geloven N, de Waal MWM, Drewes YM, et al. Deprescribing preventive cardiovascular medication in patients with predicted low cardiovascular disease risk in general practice-the ECSTATIC study: a cluster randomised non-inferiority trial. BMC Med. 2018;16:5.

23. Schuling J, Gebben H, Veehof LJ, Haaijer-Ruskamp FM. Deprescribing medication in very elderly patients with multimorbidity: the view of Dutch GPs. A qualitative study. BMC Fam Pract. 2012;13:56.

24. Goyal P, Anderson TS, Bernacki GM, Marcum ZA, Orkaby AR, et al. Physician perspectives on deprescribing cardiovascular medications for older adults. J Am Geriatr Soc. 2020;68:78-86.

25. Muth C, Uhlmann L, Haefeli WE, Rochon J, van den Akker M, et al. Effectiveness of a complex intervention on Prioritising Multimedication in Multimorbidity (PRIMUM) in primary care: results of a pragmatic cluster randomised controlled trial. BMJ Open. 2018;8:e17740. 
26. Schäfer I, Kaduszkiewicz H, Mellert C, Löffler C, Mortsiefer A, et al. Narrative medicine-based intervention in primary care to reduce polypharmacy: results from the cluster-randomised controlled trial MultiCare AGENDA. BMJ Open. 2018;8:e17653.

27. Engagement $P$. Technical series on safer primary care. Geneva: World Health Organization; 2016.

28. Pickering AN, Hamm ME, Dawdani A, Hanlon J, Thorpe C, et al. Older patient and caregiver perspectives on medication value and deprescribing: a qualitative study. J Am Geriatr Soc. 2020;6:746-53.
29. Cox K, Britten N, Hooper R, White P. Patients' involvement in decisions about medicines: GPs' perceptions of their preferences. Br J Gen Pract. 2007;57:777-84.

30. Protheroe J, Nutbeam D, Rowlands G. Health literacy: a necessity for increasing participation in health care. Br J Gen Pract. 2009;59:721-3.

31. von Buedingen F, Hammer MS, Meid AD, Müller WE, Gerlach FM, et al. Changes in prescribed medicines in older patients with multimorbidity and polypharmacy in general practice. BMC Fam Pract. 2018;19:131 